\title{
Der "Solifonds" - Ein Modell der Zusammenarbeit Zwischen Arbeiterbewegung und Entwicklungsbewegung
}

Le "Solifonds" - Un modèle de coopération entre le mouvement des travailleurs et celui du développement

Karl Aeschbach

\section{OpenEdition}

Journals

\section{Electronic version}

URL: http://journals.openedition.org/sjep/954

DOI: $10.4000 /$ sjep.954

ISSN: 1663-9677

Publisher

Institut de hautes études internationales et du développement

\section{Printed version}

Date of publication: 31 août 1982

Number of pages: $177-180$

ISSN: 1660-5926

\section{Electronic reference}

Karl Aeschbach, "Der "Solifonds" - Ein Modell der Zusammenarbeit Zwischen Arbeiterbewegung und Entwicklungsbewegung », Schweizerisches Jahrbuch für Entwicklungspolitik [Online], 2 I 1982, Online erschienen am: 24 Januar 2013, abgerufen am 08 September 2020. URL : http:// journals.openedition.org/sjep/954;DOI : https://doi.org/10.4000/sjep.954 


\title{
DER “SOLIFONDS" - EIN MODELL DER ZUSAMMENARBEIT ZWISCHEN ARBEITERBEWEGUNG UND ENTWICKLUNGSBEWEGUNG
}

\author{
Karl Aeschbach
}

Sekretär des SGB

\section{LE "SOLIFONDS" - UN MODELE DE COOPERATION ENTRE LE MOUVEMENT DES TRAVAILLEURS ET CELUI DU DEVELOPPEMENT}

Résumé : Après trois ans de discussions, I'USS, le PSS, I'OSED et dix autres organisations d'entraide et de développement, ont décidé à l'automne 1982 de créer le "Fonds de solidarité pour la lutte de libération sociale dans le Tiers Monde". Le Solifonds doit permettre au mouvement ouvrier de se joindre aux organisations de développement dans leur action en faveur des mouvements de revendication sociale dans le Tiers Monde. Un tel rassemblement est unique en Europe.

Le Solifonds s'orientera principalement vers la défense des droits de l'homme, des droits politiques et syndicaux. II pourra intervenir dans des actions de solidarité syndicale et politique, qui ne sont pas appuyées par les pouvoirs publics, à cause de leur caractère spécifique. La décision d'entreprendre des actions doit être approuvée par les trois quarts des membres du Conseil de Fondation, qui compte treize membres. Les décisions doivent donc découler d'un large consensus au sein du Conseil. Le Solifonds ne veut pas être en concurrence avec la solidarité syndicale telle qu'elle s'exprime actuellement, mais veut plutôt jouer un rôle de complément.

Les actions entreprises par le Solifonds doivent se concevoir à court terme, et de manière spontanée. Les projets qui demandent une conception à long terme sont du ressort traditionnel des organisations de solidarité.

Im Herbst 1982 wird die offizielle Gründung des "Solidaritätsfonds für den sozialen Befreiungskampf in der Dritten Welt" (Kurzformel "Solifonds") erfolgen. Nach einer rund dreijährigen Diskussions- und Vorbereitungsphase finden sich damit der Schweizerische Gewerkschaftsbund (SGB), die Sozialdemokratische Partei der Schweiz (SPS), das Schweizerische Arbeiterhilfswerk $(\mathrm{SAH})$ und zehn weitere Hilfswerke und entwicklungspolitische Organisationen zusammen. Der "Solifonds" soll eine engere Zusammenarbeit zwischen der traditionellen Arbeiterbewegung mit ihren mitgliederstarken Organisationen und der entwicklungspolitischen Bewegung verwirklichen, 
um gemeinsame Ziele der sozialen Befreiung der Menschen in der Dritten Welt zu verfolgen.

In der Schweiz gibt es seit mehr als zehn Jahren eine entwicklungspolitische Bewegung, die zunehmend an Bedeutung und Kontinuität in ihrer Arbeit gewonnen hat. Aber die fehlenden Beziehungen zu den Gewerkschaften und ihrer Massenbasis stellten einen grossen Mangel dar. Andrerseits müssen sich die Gewerkschaften - die sich selbst als Befreiungsbewegung der Arbeitnehmer verstehen - eingestehen, dass es ihnen ebenfalls kaum gelungen ist, ihren Mitgliedern ein Bewusstsein der Probleme der Ausbeutung in der Dritten Welt zu vermitteln, also der Idee der internationalen Solidarität eine weltweite Dimension zu verleihen.

In dieser Situation bildet die Gründung des "Solifonds" einen hoffnungsvollen Versuch, gemeinsame Ziele mit gemeinsamen Mitteln anzustreben. Eine institutionelle Verbindung dieser Art existiert unseres Wissens bisher in keinem anderen europäischen Land. Der "Solifonds" stellt ein Experiment dar, dass sich nun in der Praxis bewähren muss. Seine Urheber hoffen allerdings, dass sie auf diese Weise der Solidaritätsarbeit für die Unterdrückten und Verfolgten in der Dritten Welt eine neue Perspektive öffnen können.

\section{Die Ziele des "Solifonds"}

Der "Solifonds" hat nach dem Wortlaut des Zweckartikels der Stiftungsurkunde zum Ziel, "den sozialen Befreiungskampf in der Dritten Welt und den Kampf und die Erringung und Gewährleistung der Menschenrechte, insbesondere der politischen und gewerkschaftlichen Grundrechte in der ganzen Welt zu unterstützen sowie darüber in der Schweiz zu informieren".

Im Vordergrund steht der Kampf um die soziale Befreiung der Völker in der Dritten Welt. Damit knüpft der "Solifonds" einerseits an die historischen Erfahrungen der Arbeiterbewegung an, die in den Industrieländern im 19. Jahrhundert ebenfalls den Kampf um die Anerkennung ihrer Organisationen und die Respektierung der demokratischen Grundrechte führen musste. Andrerseits entspricht diese Zielsetzung der neueren Diskussion in entwicklungspolitischen Kreisen. Die Erfahrungen mit einem Vierteljahrhundert der "Entwicklungshilfe" haben gezeigt, dass technische und finanzielle Hilfe nicht genügt, wenn nicht gleichzeitig gerechtere gesellschaftliche Strukturen geschaffen werden. Der Slogan "Entwicklung durch Befreiung" bringt zum Ausdruck, dass man sich von der Unterstützung von Basisorganisationen der Arbeiter und Bauern in der Dritten Welt direktere Impulse erhofft. Die beteiligten Hilfswerke sehen darin eine wertvolle Ergänzung ihrer bisherigen Entwicklungsprojekte.

Wenn also die Probleme der Dritten Welt eindeutig im Vordergrund stehen, so soll doch beim Kampf um die allgemeinen Menschenrechte keine geographische Einschränkung gelten. Der "Solifonds" will damit bewusst die 
Gefahr der Einseitigkeit vermeiden. Schliesslich wird der Aufklärung der eigenen Bevölkerung über die Hintergründe und Zusammenhänge solcher Entwicklungen eine bedeutende Rolle zugemessen. Ein Teil der Spenden wird von Anfang an für Informationsarbeit eingesetzt werden.

Die Zielsetzung ist in der Stiftungsurkunde sehr weit gefasst. Es wird Aufgabe des Stiftungsrates sein, die einzelnen Aktionen auszuwählen. Dabei geht es in erster Linie um politische oder gewerkschaftliche Solidaritätsaktionen, für die in der Regel wegen ihres ausgeprägten Charakters keine öffentlichen Mittel zur Verfügung stehen. Durch die Zusammenfassung aller Kräfte erhofft man sich eine wirksame Unterstützung für den Kampf von Befreiungsbewegungen, Gewerkschaften oder anderen Basisorganisationen in der Dritten Welt.

\section{Prinzipien der Zusammenarbeit}

Die Träger des "Solifonds" sind sich bewusst, dass eine solche Zusammenarbeit ein hohes Mass an Uebereinstimmung voraussetzt. Der Fonds wird sich denn auch vorwiegend auf einige grössere Sammelaktionen pro Jahr beschränken, mit denen er sich an die Mitglieder aller beteiligten Organisationen wendet. Beschlüsse über Aktionen bedürfen der Zustimmung von drei Vierteln der Mitglieder des Stiftungsrates. Dieser umfasst dreizehn Mitglieder, wovon die drei Gründungsstifter SGB, SPS und SAH je drei, die übrigen als Mitstifter beteiligten Organisationen gemeinsam vier Vertreter stellen.

Praktisch bedeutet dies, dass Entscheidungen des Stiftungsrates weitgehend im Konsens erfolgen sollen. Wesentlich ist dabei, dass die verschiedenartigen internationalen Kontakte der Beteiligten zu einem Erfahrungsaustausch führen. In diesem Zusammenhang ist die recht breite Vielfalt der Mitstifter von Interesse, die Hilfswerke (Christlicher Friedensdienst, Terre des Hommes Schweiz), verschiedene Solidaritätskomitee (SKAAL, Nicaragua-Salvador, Anti-Apartheid-Bewegung, Saharaouis) und weitere entwicklungspolitische Organisationen umfasst (Erklärung von Bern, Arbeitsgruppen für Entwicklungspolitik, Vereinigung der Dritt-Welt-Läden).

Wesentliche Bedeutung wird aber vor allem den Beziehungen zu den internationalen Gewerkschaftsorganisationen, insbesondere zu den Berufssekretariaten des IBFG, zugemessen. Diese sollen vor jeder Beschlussfassung über eine Aktion konsultiert werden. Der "Solifonds" will keine Konkurrenz zur bestehenden internationalen gewerkschaftlichen Solidarität bilden, sondern vielmehr ergänzend tätig werden - sei es, dass er zusammen mit internationalen Gewerkschaften einen bedeutenden Streik oder eine andere Aktion unterstützt, sei es, dass er dort tätig wird, wo noch Lücken im Netz der internationalen Zusammenarbeit bestehen. Dabei ergibt sich aus der Zielsetzung, dass der "Solifonds" mit Partnern zusammenarbeiten will, die frei- 
heitliche und demokratische Ideale verfolgen.

\section{Ein Instrument des Engagement}

Der "Solifonds" bildet einen Versuch; jenen engagierten Menschen in der Schweiz, die sich mit der sozialen Befreiung in der Dritten Welt solidarisieren wollen, ein geeignetes Instrument zur Verfügung zu stellen. Dabei geht es nicht einfach darum, noch einen zusätzlichen Fonds zu schaffen, sondern vorhandene Kräfte zusammenzufassen. Jede der beteiligten Organisationen hat ihre spezifische Aufgabe : als politische oder gewerkschaftliche Massenorganisation, als operatives Hilfswerk oder als Solidaritätskomitee, das sich für die Benachteiligten in einem Land oder einer bestimmten Region besonders intensiv einsetzt.

Diese Eigenaktivitäten sollen auch künftig fortgesetzt werden. Aber dort, wo gemeinsame Ziele offenkundig werden, wollen die beteiligten Organisationen gemeinsam an die Oeffentlichkeit treten. Dabei stehen wohl kurzfristige, spontane Aktionen im Vordergrund, die sich über einige Wochen oder Monate erstrecken. Längerfristige Aufbauprojekte gehören dagegen in den traditionellen Aufgabenbereich der Hilfswerke.

Der "Solifonds" wird sich an die einzelnen Mitglieder und Sympathisanten seiner Trägerorganisationen richten. Bei vielen von ihnen ist der Wille zur gezielten Unterstützung politischer oder gewerkschaftlicher Solidaritätsaktionen vorhanden, bei anderen kann er durch geeignete Information geweckt werden. Den meisten Menschen fehlt heute die Möglichkeit, direkt und persönlich Hilfe zu leisten. Ihnen soll der "Solifonds" ein Instrument des Engagements zugunsten jener bieten, die unter Bedingungen der Ausbeutung und Unterdrückung für ihre soziale Befreiung kämpfen. 